\title{
Decrease in Growth Cone-Neurite Fasciculation by Sensory or Motor Cells in vitro Accompanies Downregulation of Aplysia Cell Adhesion Molecules by Neurotransmitters
}

\author{
Nadja Peter, Binyamin Aronoff, Fang $\mathrm{Wu}$, and Samuel Schacher \\ Center for Neurobiology and Behavior, Columbia University College of Physicians and Surgeons, and New York State \\ Psychiatric Institute, New York, New York 10032
}

Cell adhesion molecules play important roles in axon guidance and synapse formation. Recent studies suggest that the expression of some of these molecules can be regulated either by electrical activity or by specific neurotransmitters. The expression of neural cell adhesion molecule (NCAM)like molecules in Aplysia, designated apCAM, is downregulated from the surface of sensory neurons by $5-\mathrm{HT}$, a transmitter known to evoke long-term changes in the structure and function of these neurons. We tested whether the distribution of apCAM on the surface of other neurons can be regulated by treatments with other neurotransmitters known to evoke long-term functional and structural changes in Aplysia neurons, and we examined the consequences of treatments with the neurotransmitters on the pattern of growth cone-neurite interactions. We report that applications of the neuropeptide Phe-Met-Arg-Phe-amide (FMRFamide) that evoke long-term synaptic depression also reduce apCAM expression on the surface of motor cell L7 via a mechanism that appears to be similar to the mechanism mediating the 5-HT-induced change in the sensory cells. Specific treatments that affect apCAM distribution on the surface of their respective cells, 5-HT on sensory cells or FMRFamide on motor cell L7, mimic treatment with monoclonal antibodies against apCAM by evoking a significant reduction in the fasciculation of growth cones with other neurites extending from homologous cells. Thus, one way that activity-dependent mechanisms might influence cell-cell interactions during development, regeneration, or following stimuli that evoke structural changes in the mature nervous system is via the release of neurotransmitters or neuropeptides that have multiple actions on receptive cells, including the regulation of expression or distribution of cell adhesion molecules.

[Key words: cell adhesion molecules, growth cones, fasciculation, FMRFamide, 5-HT, neurite outgrowth, Aplysia californica]

The guidance of axons and the wiring of the nervous system are facilitated by the actions of diffusible substances from intermediatc or final targets (Lumsden and Davies, 1983; Tessier-

Received June 29, 1993; revised Aug. 20, 1993; accepted Aug. 26, 1993

We thank Drs. J. Koester and I. Kupfermann for their comments on earlier drafts of the manuscript, and Robert Woolley for technical assistance. This work was supported by NIH Grant NS27541.

Correspondence should be addressed to Samuel Schacher, Ph.D., Center for Neurobiology and Behavior, Columbia University College of Physicians and Surgeons, 722 West 168th Street, New York, NY 10032.

Copyright (C) 1994 Society for Neuroscience $0270-6474 / 94 / 141413-09 \$ 05.00 / 0$
Lavigne et al., 1988; O'Leary et al., 1991) or by appropriate interactions via specific cell or substrate adhesion molecules (for reviews, see Jessell, 1988; Rutishauser et al., 1988; Bixby and Harris, 1991; Edelman and Cunningham, 1991; Reichardt and Tomaselli, 1991; Takeichi, 1991; Grenningloh and Goodman, 1992). In addition, both diffusible and membrane-associated molecules can also evoke regressive responses in growth cones, thereby affecting the trajectory of axons (Haydon et al., 1984; Caroni and Schwab, 1988; Cox et al., 1990; Raper and Kapfhammer, 1990; Oakley and Tosney, 1991; for review, see Schwab et al., 1993).

There is evidence that extracellular matrix, cell adhesion, or diffusible molecules may also affect later stages of development or axon regeneration, including the formation and maintenance of chemical synapses. For example, secreted molecules and deposition of various extracellular matrix molecules in the basal lamina surrounding muscle cells are critical for the development of neuromuscular junctions and the reestablishment of synaptic contacts at preexisting end-plate zones following denervation (Usdin and Fischbach, 1986; Hunter et al., 1989; McMahon, 1991; Campanelli et al., 1991; Hall and Sanes, 1993). The branching and patterns of connectivity of neurons can be altered by changing neural cell adhesion molecule (NCAM) expression or extent of glycosylation (Fraser et al., 1984; Tang et al., 1992). In addition, diffusible substances derived from local targets may initiate growth from shafts of preexisting axons (O'Leary et al., 1991). In some instances the regulation of the critical molecules can be accomplished via electrical activity (Landmesser et al., 1990). The mechanisms by which this activity influences the expression and distribution of developmentally relevant molecules as well as the signal transduction machinery affecting subsequent changes in cell-cell intcractions remain poorly understood.

The neurons of Aplysia, both during development and in the mature animal, express relatively high levels of membrane glycoproteins, designated apCAMs, that have structural homology with vertebrate NCAM and Drosophila fasciclin II (Keller and Schacher, 1990; Schacher et al., 1991; Mayford et al., 1992). Antibodies recognizing extracellular epitopes of apCAM evoke defasciculation of extending neurites from many Aplysia neurons (Keller and Schacher, 1990). Recent studies indicate that 5-HT, an important transmitter in behavioral sensitization of various defensive withdrawal reflexes in Aplysia (Glanzman et al., 1989; Mackey et al., 1989; Zhang et al., 1991), can alter the synthesis of apCAM (Barzilai et al., 1989) and its distribution on the surface of adult sensory neurons (SNs) mediating the reflexes (Mayford et al., 1992). Treatments with 5-HT down- 

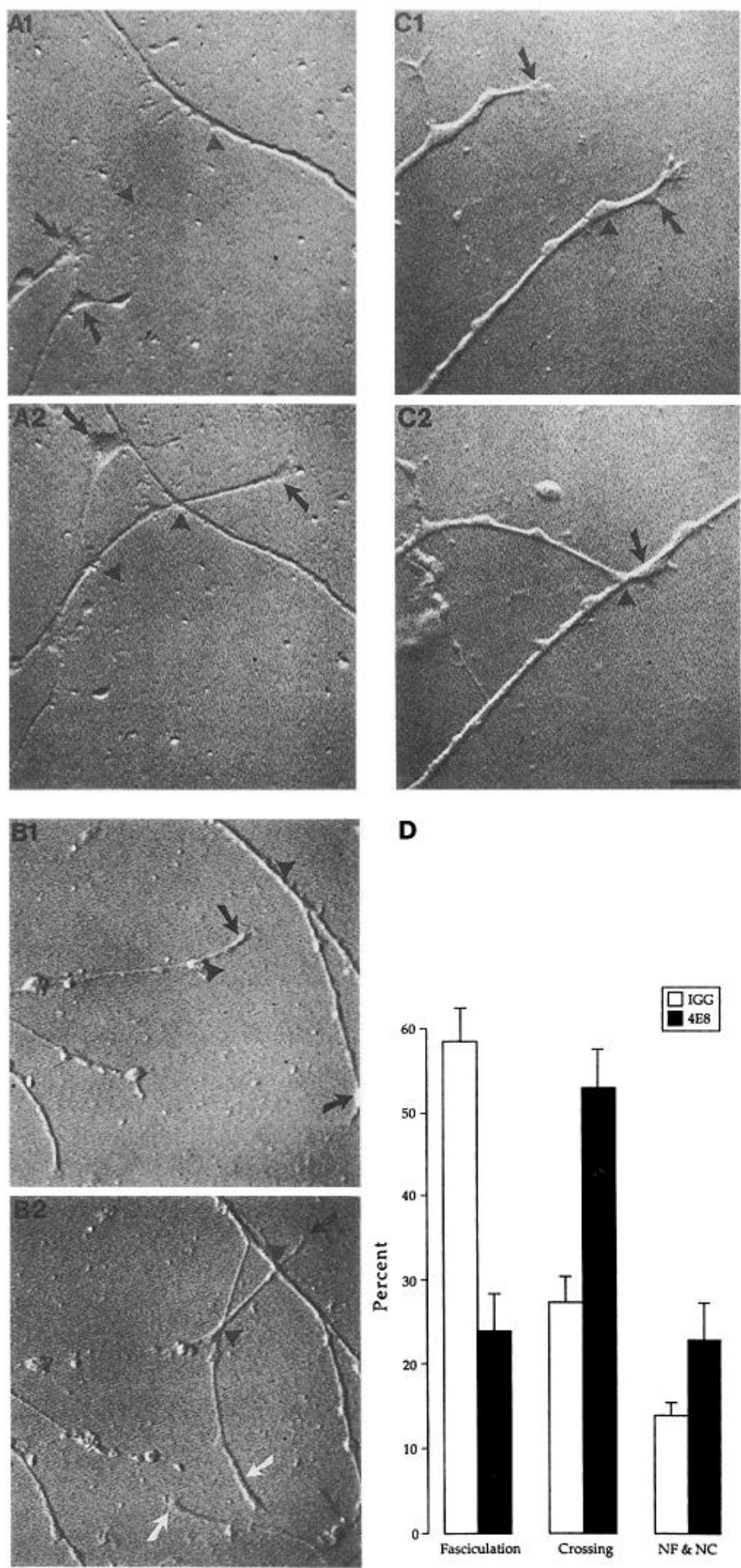

D

Figure 1. Anti-apCAM antibody reduces fasciculation of $\mathrm{SN}$ growth cones on SN neurites. $A$ and $B$, Nomarski contrast micrographs of SN growth cones and neurites before $(A 1$ and $B 1)$ and after $(A 2$ and $B 2)$ incubation with anti-apCAM mAb 4E8. Two growth cones in $A$ (arrows) extend and cross (arrowheads) other sensory neurites. In $B$, one growth cone (arrow, upper right) extends and crosses another sensory neurite (arrowhead, upper right) while a second growth cone (arrow, lower right) fails to fasciculate or to cross. Two new growth cones appear at the bottom of $B 2$ (white arrows). One crosses a preexisting sensory neurite (arrowhead, center). $C$, Micrographs of SN growth cones and neurites before $(\mathrm{Cl})$ and after $(\mathrm{C2})$ incubation with a control mouse antibody (IgG). A growth cone in $\mathrm{Cl}$ (top arrow) fasciculates (arrow in $C 2$ ) with the other sensory neurite (arrowhead). Scale bar, $25 \mu \mathrm{m}$. $D$, Summary of the effects of treatments on behavior by SN growth cones. Each bar is the mean \pm SEM of the percent of growth cones per culture dish responding to treatment with a given behavior. An overall ANOVA indicated a significant effect of treatment $(\mathrm{df}=2,20, F=21.503, p<$ regulate the level of apCAM from the surface of SNs by increasing membrane retrieval via endocytosis (Bailey et al., 1992b). Treatments with 5-HT also induce long-term enhancement of the SN-L7 synapse in vitro (Montarolo et al., 1986), including the formation of new sensory cell branches and presynaptic varicosities with transmitter release sites (Glanzman et al., 1991; Schacher et al., 1991; Bailey et al., 1992c). One mechanism by which regulating the expression of apCAM might affect the structural changes accompanying synaptic facilitation is that the downregulation of apCAM on the surface of sensory neurites, with no apparent effect on apCAM levels on the motor neurites and axons, could reduce $\mathrm{SN}-\mathrm{SN}$ neurite interactions and facilitate interactions between sensory processes and adjacent motor neurites. This in turn might facilitate the formation of new transmitter release sites between SNs and motor cells. A parallel mechanism involving the regulation of apCAM may contribute to structural changes in SNs accompanying long-term synaptic depression. The inhibitory transmitter Phe-Met-ArgPhe-amide (FMRFamide) evokes both functional and structural changes at the SN-L7 synapse, including the retraction of sensory neurites and a decrease in the number of presynaptic varicosities (Belardetti et al., 1987; Montarolo et al., 1988; Schacher and Montarolo, 1991). Because apCAM is enriched at sites of synaptic contact (Keller and Schacher, 1990), it is possible that transmitter-induced alteration in the distribution of apCAM on the surface of the postsynaptic motor neurons, especially at the release sites, may contribute to the structural changes accompanying long-term inhibition that are evoked by FMRFamide.

One consequence of the transmitter-induced downregulation of apCAM molecules from the surface of the cells would be an alteration in the behavior of growth cones when they interact with the neurites of homologous cells. We report here that applications of 5-HT or FMRFamide, known to evoke opposite forms of long-term structural and functional changes of the $\mathrm{SN}-$ L7 synapse in vitro, alter the levels of apCAM on the surface of either the sensory cell (5-HT) or motor cell (FMRFamide), respectively, and decrease the fasciculation of their respective growth cones with neurites regenerated from cell homologs. The transmitter actions are cell specific in that over the same time course 5-HT has no significant effect on motor cells and FMRFamide has no effect on sensory cells, in terms of both apCAM distribution and growth cone-neurite interactions.

\section{Materials and Methods}

Cell culture and treatments. Mechanosensory cells located in the pleural ganglion and gill and siphon motor cell L7 in the abdominal ganglion were isolated from adult $(80-100 \mathrm{gm})$ or juvenile $(1-3 \mathrm{gm})$ animals, respectively, (Howard Hughes Medical Institute Mariculture Facility at the University of Miami, FL) and maintained in culture as described previously (Schacher and Proshansky, 1983; Rayport and Schacher, 1986; Montarolo et al., 1988). Individual sensory cells or motor cells in culture for $4 \mathrm{~d}$ were used to determine the effects of transmitter applications known to evoke long-term modulation of sensorimotor connections. Cells were treated with $5 \times 5 \mathrm{~min}$ applications of $5 \mu \mathrm{M}$ 5 -HT or $1 \mu \mathrm{M}$ FMRFamide in perfusion medium consisting of $50 \%$ Leibowitz L15 medium modified with additional salts plus 50\% seawater during a $2 \mathrm{hr}$ period (Montarolo et al., 1986, 1988). The distribution of apCAM was determined by treating cells with FITC-labeled anti-apCAM monoclonal antibodies (mAbs) $(10 \mu \mathrm{g} / \mathrm{ml}$ FITC-4E8) applied for $20 \mathrm{~min}$ before initial photographs and treatment (Keller and

$0.001)$. Treatment with $4 \mathrm{E} 8$ affected the proportions for each observed behavior $(\mathrm{df}=1,10 ; p<0.001$ for fasciculation; $p<0.002$ for crossing; $p<0.04$ for failure to interact or NF and NC). 


\section{SN Control}
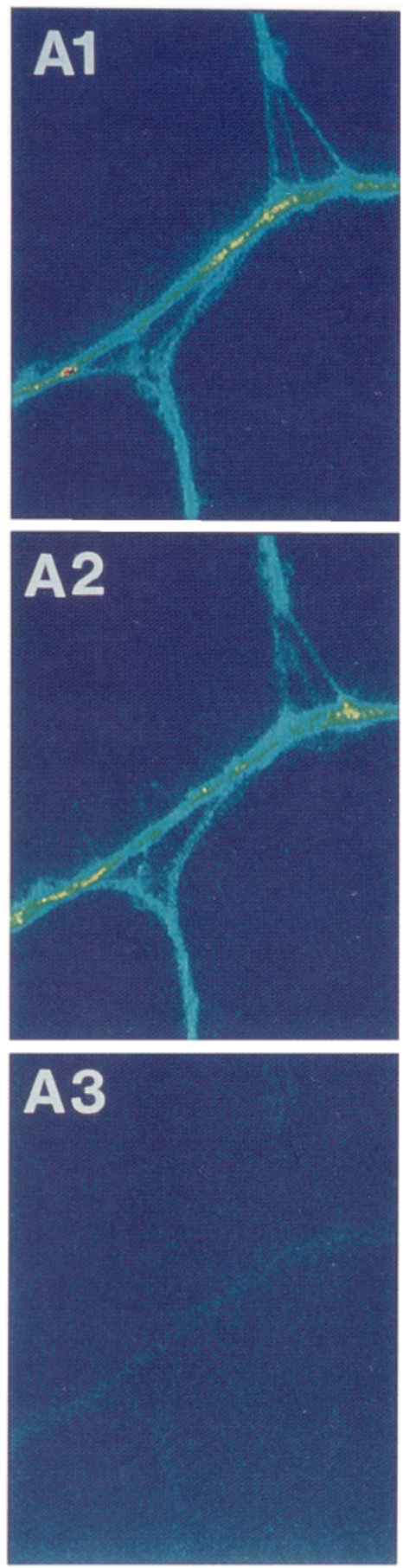

\section{SN FMRF}
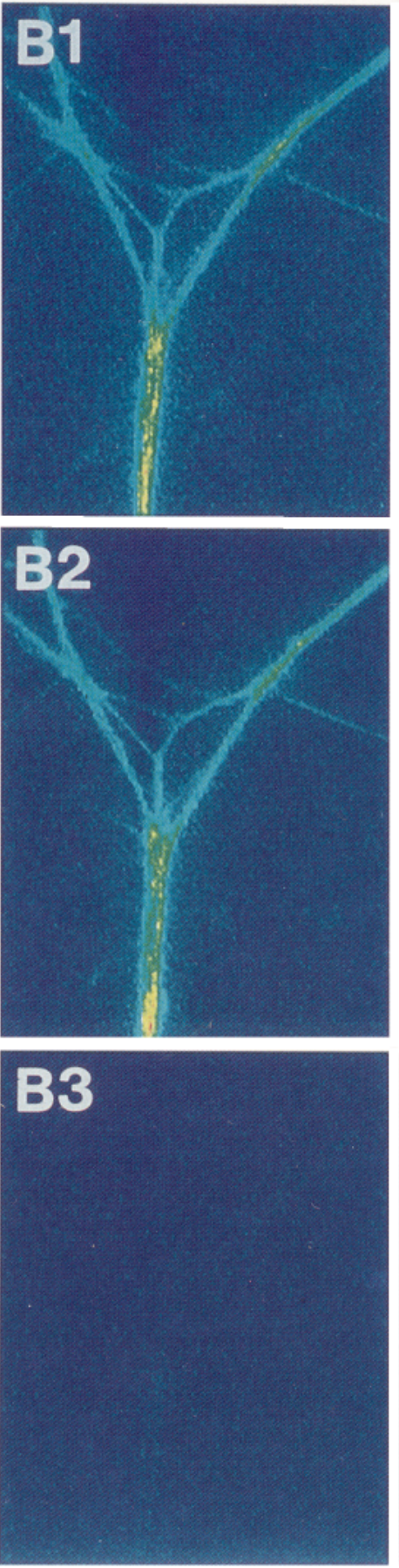
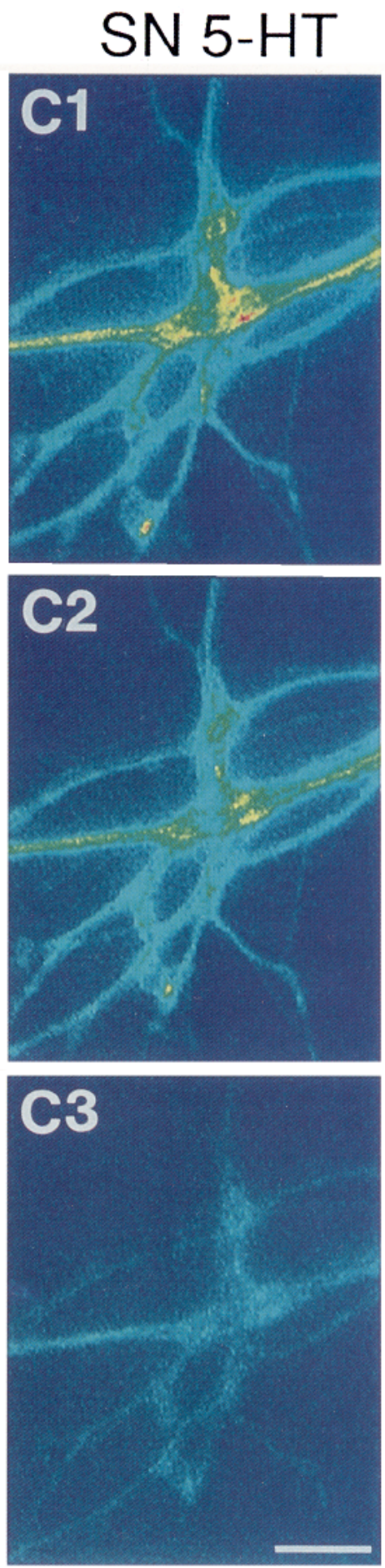

Figure 2. 5-HT, but not FMRFamide, downregulates ApCAM expression on sensory neurites: fluorescent images of the distribution of FITC4E8. Micrographs are pseudocolor representation of 4E8-apCAM distribution (red, high intensity blue, low intensity). A1-A3, Control treatments $(2 \mathrm{hr})$ evoke little change in the overall distribution of FITC-4E8 on the surface of the sensory neurites $(A 1$ and $A 2)$. Since most of the fluorescence is quenched by exogenous $\mathrm{CuSO}_{4}(A 3)$, it appears that little of the antibody-tagged apCAM is internalized during control treatment. $B 1-B 3$, FMRFamide applications evoke little change in the overall distribution and intensity of FITC-4E8 (BI and $B 2$ ). Most of the staining is quenched by exogenous $\mathrm{CuSO}_{4}$, suggesting little internalization with treatment $(B 3)$. $C 1-C 3$, Treatment with repeated applications of 5-HT alters the distribution and intensity of FITC-4E8 on sensory neurites. Note that high intensity staining (red and bright yellow) has been abolished (compare $C 1$ and $C 2$ ). The failure to abolish a substantial level of staining with $\mathrm{CuSO}_{4}(C 3)$ suggests that a significant portion of the FITC-4E8-apCAM has been internalized with treatment. Scale bar, $25 \mu \mathrm{m}$.

Schacher, 1990; Mayford et al., 1992). After rephotographing FITC4E8-apCAM distribution 15-30 min following last control or transmitter application, perfusion medium containing $0.25 \mathrm{~mm} \mathrm{CuSO}_{4}$ was added for 5 min to quench the fluorescent signal from the surface of the cells (Rajarathnam et al., 1989), and the same view areas of the labeled neurites were photographed for a third time.

The effects of anti-apCAM (4E8) and control IgG antibodies $(200 \mu \mathrm{g}$ / $\mathrm{ml}$ for $3 \mathrm{hr}$; Keller and Schacher, 1990) or the repeated applications of 


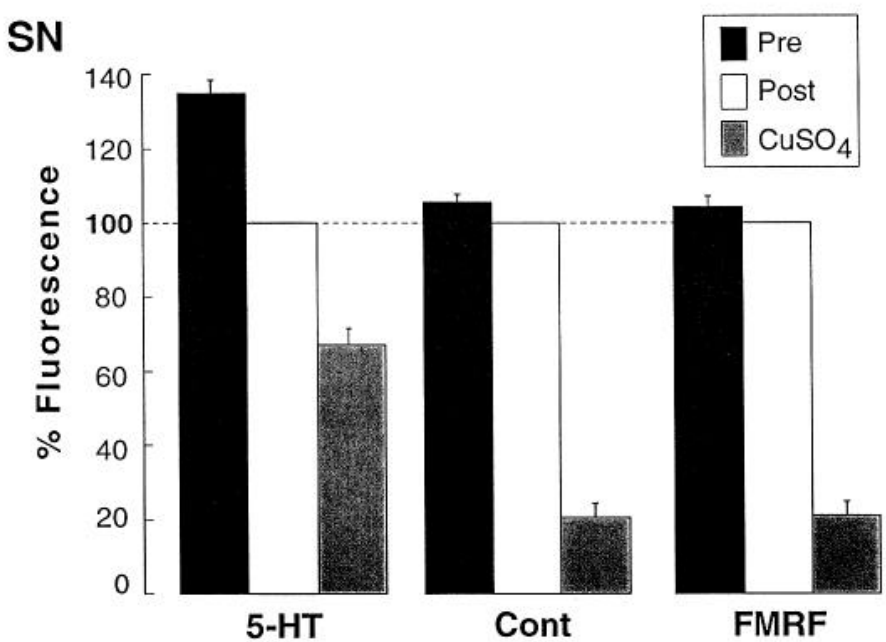

Figure 3. Changes in FITC-4E8-apCAM distribution on SN neurites by $5-\mathrm{HT}$. The bars are the mean \pm SEM of the intensity of bound FITC4E8 normalized for each cell to the level obtained after treatment (Post). An ANOVA indicated a significant effect of treatment on staining intensity $(\mathrm{df}=2,12, F=35.203, p<0.001)$ and on level of fluorescence insensitive to quenching $(F=45.209, p<0.001)$. Multicomparison $t$ tests (Dunnett's $t$ ) indicated that treatment with 5-HT had a significant effect on the pixel intensity $(t=7.517, p<0.01)$ and the intensity of the component insensitive to quenching $(t=8.295, p<0.01)$ compared to control. By contrast, treatment with FMRFamide had little effect on pixel intensity $(t=0.53, p>0.5)$ or the insensitive component $(t=$ $0.107, p>0.5$ ) compared to treatment with control solutions.

5-HT or FMRFamide on growth cone-neurite interactions were examined on 2- or 3-d-old cultures. These cultures were used because the growth rate for extending neurites remains at its peak, permitting the scoring of more growth cone-neurite interactions per culture dish. Each culture contained either a single L7 motor cell or 5-10 sensory cells in close proximity. Growth cones of L7 or the sensory cells were photographed before treatment and $1 \mathrm{hr}$ after treatment (with antibodies or with neurotransmitter) and scored with a blind procedure for the type of interaction with other neurites (fasciculation, crossover, or neither behavior). Since the number of identifiable growth cones per dish varied from 5 to 15 for sensory cell cultures and from 50 to 100 per motor cell culture, each culture dish was scored as a single sample with a given distribution of behaviors for the growth cones. The frequency for each behavior per culture dish was then averaged to obtain final values.

Fluorescent and Nomarski light microscopy. Cells were photographed with a Nikon Diaphot microscope using a Dage 66 SIT camera connected to a Dell computer with a PC Vision Plus frame grabber. Fluorescent images of FITC-4E8-apCAM distribution on sensory or motor neurites were photographed before and $15-30 \mathrm{~min}$ after treatments and a third time following fluorescence quenching with $\mathrm{CuSO}_{4}$. The same areas of neurite outgrowth (six randomly selected areas per cell containing a total of 1500-3000 $\mu \mathrm{m}^{2}$ of membrane) were examined for each neuron under each condition. The change in the intensity of staining was determined by averaging the pixel intensities measured before and after treatments and addition of $\mathrm{CuSO}_{4}$ for each view area (Mayford et al., 1992). Values for each cell were then normalized to the intensity value obtained after the experimental or control treatment.

Growth cones were photographed before and $1 \mathrm{hr}$ following treatments (a total of about $4 \mathrm{hr}$ between photographs). Only growth cones that had extended sufficiently to have the potential of interacting with other neurites were included in the sample. The various treatments did not have a significant effect $(p>0.4)$ on the overall rate of neurite extension on the polylysine-coated glass substrate, which was approximately $25 \mu \mathrm{m} / \mathrm{hr}$ (range of $15-30 \mu \mathrm{m}$ for all treatments).

\section{Results}

Both anti-apCAM antibody and 5-HT alter behavior of $S N$ growth cones

Overnight treatment with anti-apCAM mAbs or their Fab fragments alters the pattern of neurite outgrowth by SNs and motor
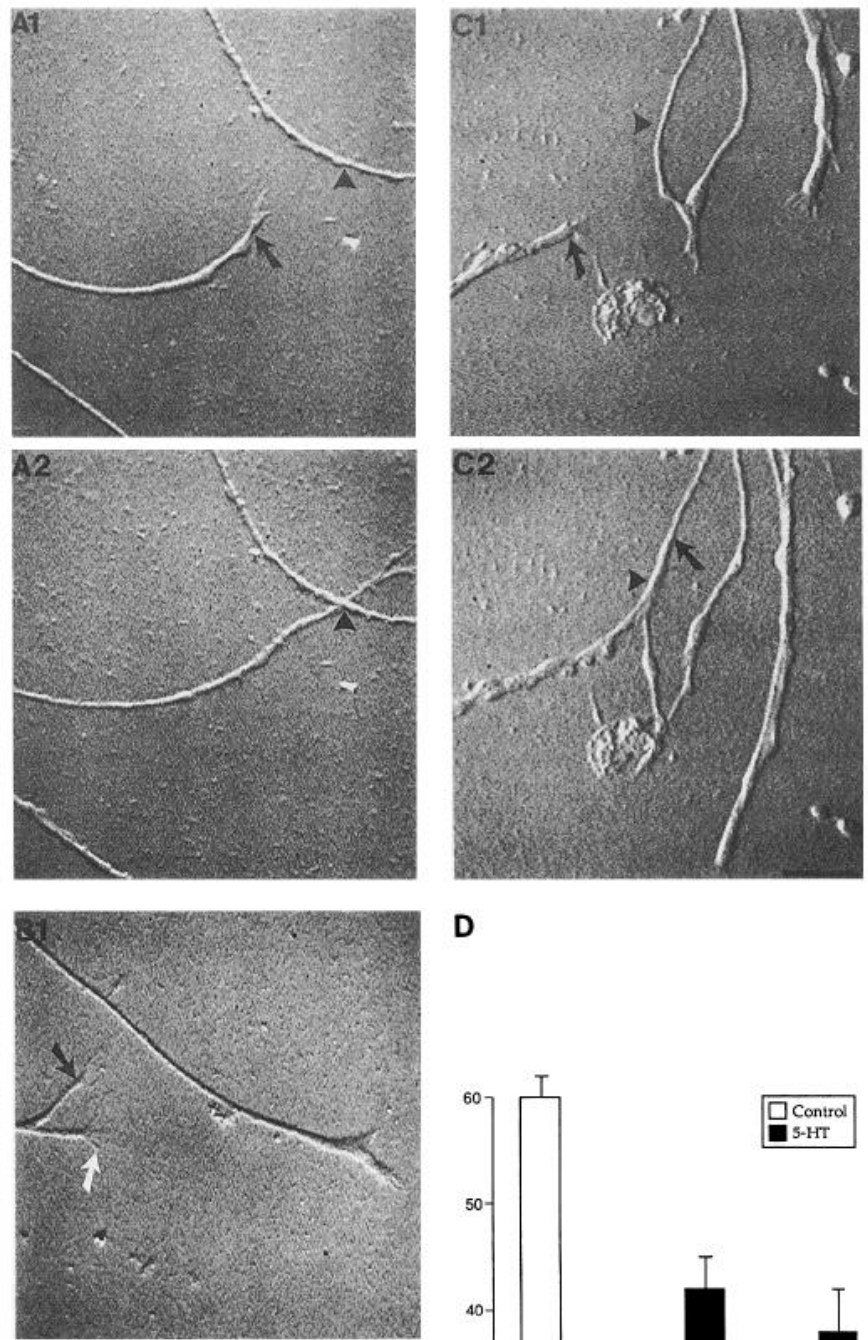

D
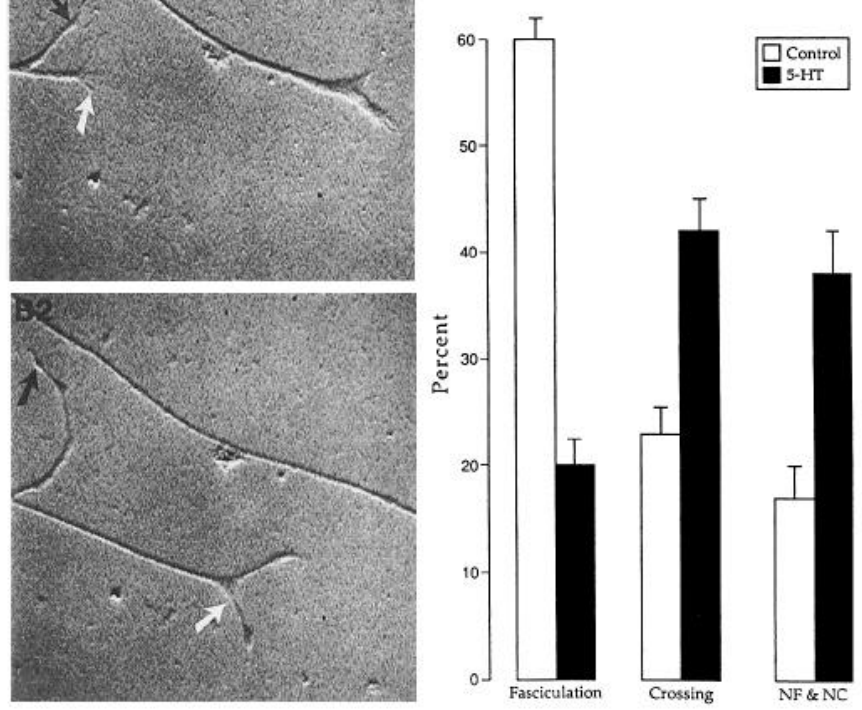

Figure 4. 5-HT reduces fasciculation of $\mathrm{SN}$ growth cones on $\mathrm{SN}$ neurites. $A$ and $B$, Micrographs of SN neurites before $(A I$ and $B I)$ and after ( $A 2$ and $B 2$ ) applications of 5-HT. A growth cone (arrow) in $A$ crosses another SN neurite (arrowhead). In B, two growth cones (black and white arrows) extend branches that fail to interact with the other SN neurites. $C$, Micrographs of SN neurites before $(C 1)$ and after $(C 2)$ treatment with control solutions. A growth cone (arrow) fasciculates with another sensory neurite (arrowhead). Scale bar, $25 \mu \mathrm{m}$. $D$, Summary of the effects of treatment on behavior of SN growth cones. Each bar is the mean \pm SEM of the percent of growth cones per culture dish responding to treatment with a given behavior. An overall ANOVA indicated a highly significant effect of treatment ( $\mathrm{df}=2,40, F=47.226$, $p<0.001$ ). Treatment with 5-HT had a significant effect on proportion of observations for each type of growth cone behavior $(\mathrm{df}=2,20 ; p<$ 0.001 for fasciculation, $p<0.001$ for crossing, $p<0.001$ for NF and NC). 
cell $\mathrm{L} 7$ by significantly increasing the number of individual neurites emerging from the cut axon stumps without affecting the overall rate of neurite extension (Keller and Schacher, 1990; Mayford et al., 1992). To evaluate whether this effect of the antibody is a consequence of changing the proportion of SN growth cones fasciculating on other $\mathrm{SN}$ neurites, we compared the behaviors of individual growth cones following treatments with anti-apCAM mAb (4E8) with growth cones of cells treated with control mouse antibodies (Fig. 1). Growth cone behaviors were separated into three categories (see also Hawver and Schacher, 1993): (1) crossing over (Fig. 1A2,B2), (2) fasciculation (Fig. 1C2), and (3) failure to fasciculate or to cross (Fig. 1B2).

Treatment with the anti-apCAM mAb 4E8 ( $N=6$ cultures, 54 growth cones) had a significant effect on the behavior of sensory cell growth cones (Fig. $1 D$ ) compared to treatment with control antibodies ( $N=6$ cultures, 49 growth cones). The frequency of growth cone fasciculation on other neurites decreased by nearly $60 \%$ (from $58.7 \% \pm 4.1 \%$ of all behaviors for treatment with control $\mathrm{IgG}$ to $24.8 \% \pm 4.4 \%$ for treatment with 4E8). By contrast, treatment with 4E8 increased crossing events by almost $90 \%$ (from $28.0 \% \pm 2.9 \%$ for control treatments to $52.7 \% \pm 4.5 \%$ ), and increased failures to interact with neurites (no fasciculation and no crossing, or NF and NC) by nearly $70 \%$ (from $13.3 \% \pm 1.4 \%$ for control treatments to $22.5 \% \pm 4.6 \%$ ).

These results are consistent with the notion that apCAM molecules on both the growth cone and neurites of SNs are blocked by antibody and can no longer undergo homophilic molecular interactions, thereby leading to a decline in fasciculation. This suggests that the removal of these molecules from the surface of the sensory cells might produce a similar phenotype: a reduction in growth cone-neurite fasciculation. Recent studies indicate that prolonged exposure to 5-HT produces a downregulation via endocytosis of apCAM from the surface of sensory cells (Bailey et at., 1992b). We therefore examined first whether repeated brief applications of 5-HT or the neuropeptide FMRFamide, transmitter treatments known to modulate the long-term functional and structural properties of $\mathrm{SN}-\mathrm{L} 7$ connections, will modulate apCAM levels on the surface of SNs, and second whether the same treatments with transmitters will alter the behavior of $\mathrm{SN}$ growth cones when contacting neurites of other SNs.

As previously reported for a single prolonged exposure to 5-HT (Mayford et al., 1992), repeated applications of 5-HT ( $N$ $=5 \mathrm{SNs})$ evoked a significant decline of $26 \%(134.8 \% \pm 3.5 \%$ to $100 \%$ ) in the FITC-apCAM signal observed in SN neurites compared to insignificant changes of about $5 \%(105.8 \% \pm 2.4 \%$ to $100 \%)$ for repeated applications of FMRFamide ( $N=5 \mathrm{SNs})$ and $3 \%(103.6 \% \pm 2.8 \%$ to $100 \%)$ for control treatments $(N=$ 5 SNs; Figs. 2, 3). Consistent with the finding that prolonged 5-HT treatment downregulates apCAM from the surface of SNs and increases the level of apCAM intracellularly by increasing the rate of endocytosis of surface membrane (Bailey et al., 1992b), repeated applications with 5-HT selectively increased the level of fluorescence apparently present in intracellular compartments. Quenching the fluorescence signals from the external surface membrane of SNs reveals a small insensitive component remaining after either control or FMRFamide treatment of $20.6 \%$ $\pm 3.9 \%$ and $21.2 \% \pm 3.7 \%$, respectively, but a larger insensitive component of $67.2 \% \pm 4.4 \%$ after 5 -HT treatment (compare Fig. $2 A 3, B 3$ to Fig. $2 C 3$; see also Fig. 3).

We also examined whether the neurotransmitters affect SN

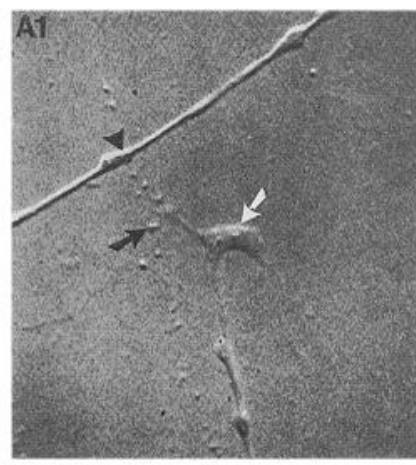

B
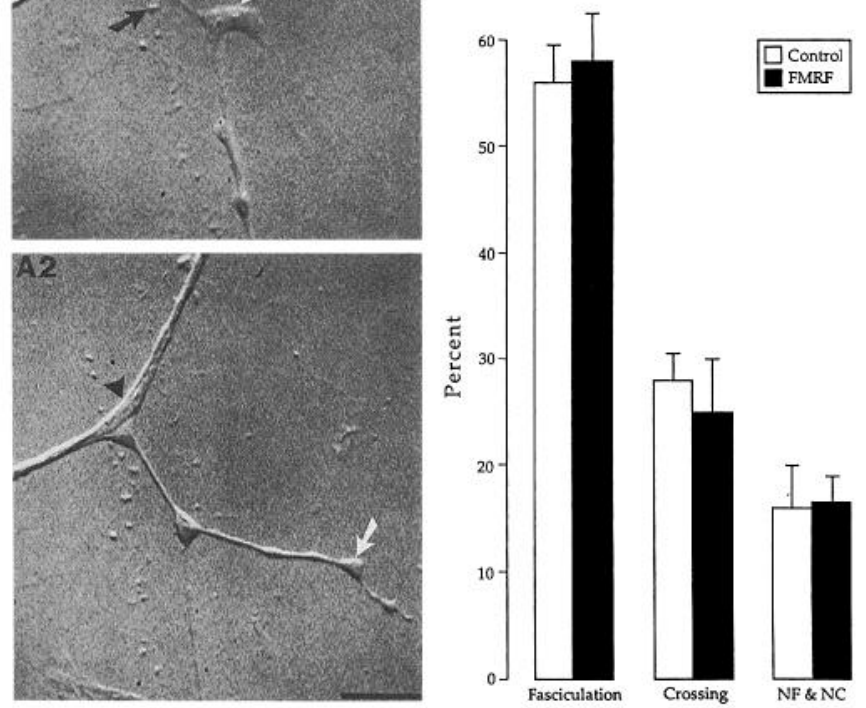

Figure 5. FMRFamide does not alter fasciculation of SN growth cones with SN neurites. $A$, SN neurites before $(A I)$ and after $(A 2)$ FMRFamide. One growth cone (black arrow, $A 1$ ) fasciculates with an SN neurite in its path (arrowhead). Another growth cone (white arrow) extends on the substrate. Note the change in the shape of the contacted neurite indicative of the tension produced by the fasciculating growth cone. Scale bar, $25 \mu \mathrm{m}$. B. Summary of the effects of treatment on behavior by $\mathrm{SN}$ growth cones. Each bar is the mean \pm SEM of the percentage of growth cones per culture dish responding to treatment with a given behavior. An overall ANOVA indicated that treatment had little effect on growth cone behavior $(\mathrm{df}=2,28, F=0.599, p>0.55)$. FMRFamide had little effect on fasciculation $(p>0.79)$, crossing $(p>0.29)$, or NF and $\mathrm{NC}(p>0.43)$.

growth cone-SN neurite interactions. The ability of a neurotransmitter to influence growth cone behavior correlated with the actions of the neurotransmitter on the distribution of apCAM on the surface of the SNs. Treatments with 5-HT that produced a significant reduction in apCAM expression on the surface of SNs altered SN growth cone behavior, as was observed with anti-apCAM mAb (compare Figs. 1, 4). Treatment with 5-HT ( $N=11$ cultures, 83 growth cones) had a significant effect on the behavior of sensory growth cones compared to treatment with control solutions ( $N=11$ cultures, 77 growth cones). The frequency of fasciculation decreased by nearly $70 \%$ (from $59.8 \%$ $\pm 2.0 \%$ for control treatments to $19.5 \% \pm 2.4 \%$ for 5 - HT applications). Conversely, treatment with 5-HT increased the frequency of growth cones crossing other neurites by $90 \%$ (43.6\% $\pm 3.1 \%$ compared to $22.9 \% \pm 2.6 \%$ for control treatments), and the frequency of failures to interact with other neurites (NF and NC) by more than $100 \%(36.6 \% \pm 4.2 \%$ compared to $17.3 \%$ $\pm 3.0 \%$ for control treatments). By contrast, the frequency of growth cone fasciculation on other $\mathrm{SN}$ neurites, as well as other behaviors, was not altered by treatments with FMRFamide (Fig. 5 ). With treatment ( $N=8$ cultures, 63 growth cones), the frequency of fasciculating, crossing, and failure to interact was $57.6 \% \pm 4.6 \%, 25.6 \% \pm 4.8 \%$, and $16.8 \% \pm 2.5 \%$, respectively. These values are comparable to those obtained for another set of cultures given control treatments $(N=8$ cultures, 68 growth 


\section{L7 Control}
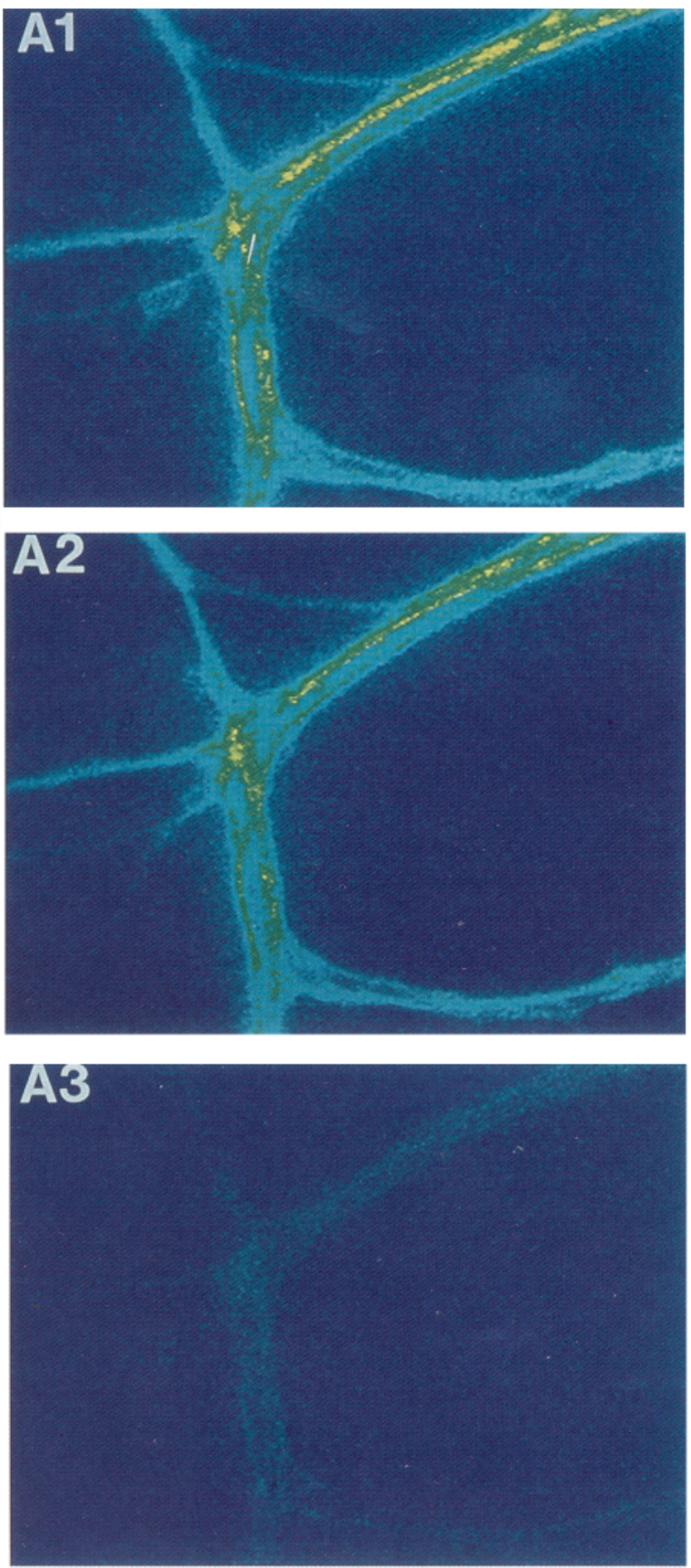

\section{L7 FMRF}
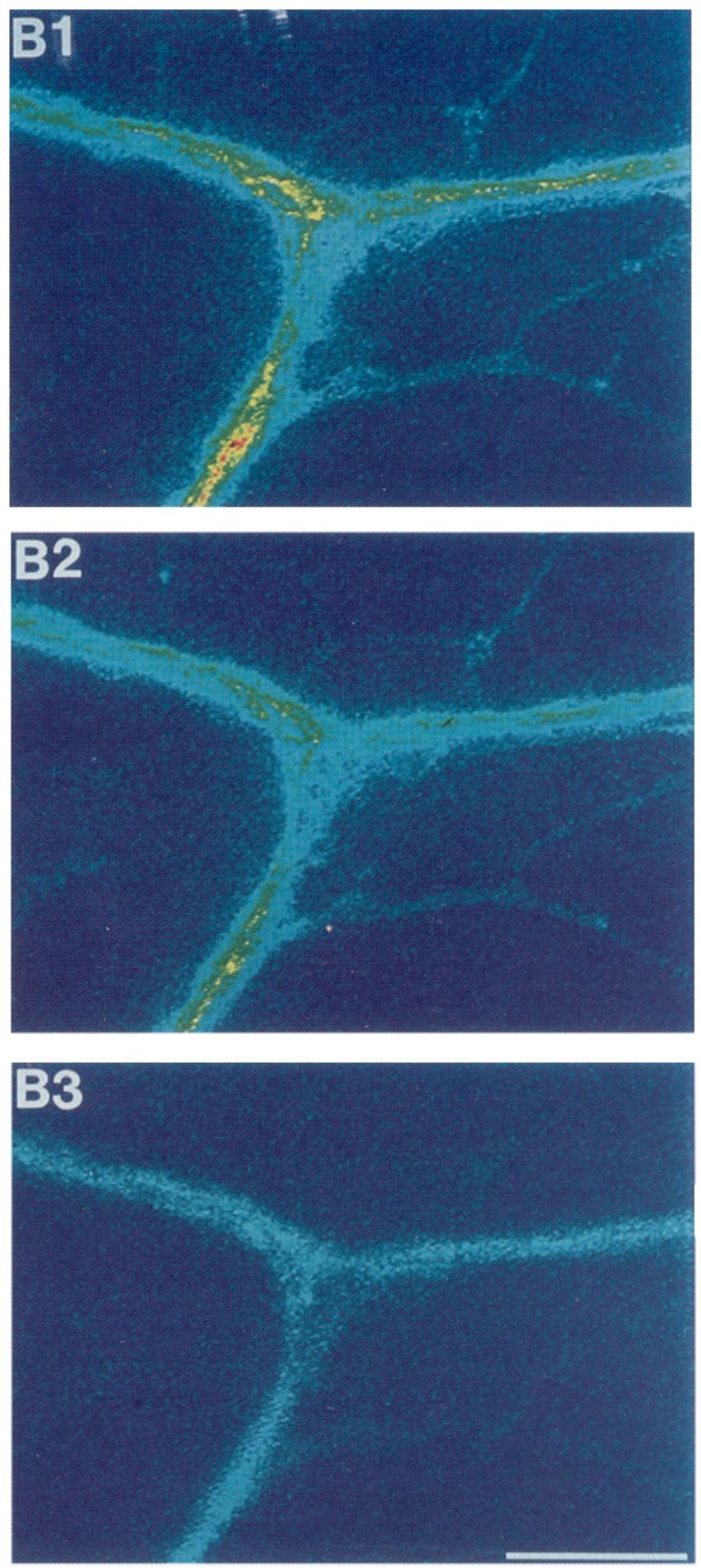

Figure 6. FMRFamide downregulates apCAM expression on neurites of motor cell L7. $A 1-A 3$, Control treatment evokes little or no change in the intensity of FITC-4E8-apCAM (compare $A I$ and $A 2$ ). Nearly all the fluorescent staining is quenched with exogenous CuSO ${ }_{4}(\mathrm{Compare} A 2$ and A3). 5-HT also evokes little change in the intensity of staining (see Mayford et al., 1992). B1-B3, Repeated applications of FMRFamide leads to a significant reduction in the intensity of FITC-4E8 staining of apCAM (compare $B 1$ and $B 2$ ). Following quenching with CuSO $\mathrm{C}_{4}$, a detectable level of staining persists (compare $B 2$ and $B 3$ ), suggesting transmitter-induced internalization of FITC-4E8 bound to apCAM. Scale bar, $25 \mu$ m.

cones): $56.1 \% \pm 3.5 \%, 28.3 \% \pm 2.6 \%$, and $15.6 \% \pm 4.4 \%$, respectively.

FMRFamide alters both distribution of ApCAM on L7 and behavior of $L 7$ growth cones

Previous studies indicated that the downregulation of apCAM by 5 -HT was cell specific. Treatment with 5 -HT had no signif- icant effect on apCAM distribution on the surface membrane of L7. We therefore examined whether FMRFamide, a transmitter capable of altering the structure of presynaptic sensory cells in the presence of the postsynaptic target cell L7 (Schacher and Montarolo, 1991), can affect the distribution of apCAM on L7, and whether this cell surface change correlates with transmitter-induced changes in the behavior of $\mathrm{L} 7$ growth cones. 
Repeated application of FMRFamide evoked a change in the distribution of FITC-anti-apCAM on the surface of L7 that paralleled the changes evoked by 5-HT on SNs. Treatment with FMRFamide significantly reduced the overall intensity of staining and increased the intensity of the fluorescent component that was insensitive to external quenching (Fig. 6). The decline in fluorescent signal with FMRFamide $(N=5 \mathrm{~L} 7 \mathrm{~s})$ was greater than $30 \%(147.0 \% \pm 7.3 \%$ to $100 \%)$, with $70.8 \% \pm 4.8 \%$ of the remaining signal insensitive to quenching (Fig. 7). By contrast, repeated applications of 5-HT had little effect on the fluorescent signal when compared to control treatment. Treatment with 5-HT $(N=5 \mathrm{~L} 7 \mathrm{~s})$ evoked a small decline in fluorescent signal of $2 \%(102.2 \% \pm 4.0 \%$ to $100 \%)$ that was not significantly different than the decline of about $5 \%(105.6 \% \pm 1.6 \%$ to $100 \%)$ observed for $\mathrm{L} 7$ treated with control solutions $(N=5)$. Upon quenching the external signal, fluorescent intensity was reduced to $23.8 \% \pm 4.6 \%$ and $21.6 \% \pm 3.9 \%$ for control and $5-\mathrm{HT}$ treatments, respectively (Fig. 7).

Treatment with FMRFamide resulted in a significant change $(\mathrm{df}=2,15 ; F=11.333, p<0.002)$ in the pattern of interaction of L7 growth cones with other neurites of L7. Repeated application of FMRFamide $(N=6)$ resulted in a decrease in the frequency of fasciculation of L7 growth cones with other L7 neurites and an increase in the frequency of crossing events. The frequency of fasciculation declined to $26.7 \% \pm 3.0 \%$ of all behaviors following FMRFamide treatment compared to $50.7 \%$ $\pm 3.9 \%$ following control treatment $(N=6 ; t=4.467, p<0.01)$. Crossing behaviors were observed to increase as reflected by the behavior of the remaining growth cones. Failures of growth cones to fasciculate or cross were rare in L7 cultures because of the high density of neurites. Correlated with the failure of 5-HT to alter FITC-labeled apCAM distribution on the neurites of L7, treatment with 5-HT had no significant effect on the pattern of outgrowth of L7 motor neurites when compared to the pattern observed with control treatments. The frequency of fasciculation of L7 growth cones on other L7 neurites was $46.5 \% \pm 4.4 \%$ following treatment with 5-HT $(N=6)$ compared to the $50.8 \%$ $\pm 3.9 \%$ observed following treatment with control solutions $(t$ $=0.806, p>0.5$ ).

\section{Discussion}

Our results indicate that the distribution of cell adhesion molecules on the surface of identified neurons of Aplysia can be modulated by the actions of specific neurotransmitters. Exposing SNs to repeated applications of 5-HT (but not FMRFamide) or exposing L7s to repeated applications of FMRFamide (but not 5-HT) produces similar changes in the distribution of FITCanti-apCAM mAbs on the surface of the cells. These results suggest that FMRFamide actions on apCAM distribution on L7 may recapitulate those produced by 5 -HT on the sensory cells: to downregulate the expression of apCAM on the surface of the neurites via endocytic retrieval of surface membrane (Bailey et al., 1992b; Mayford et al., 1992). Recent evidence suggests that the downregulation of apCAM from the surface of SNs by 5-HT may be mediated by cAMP (Bailey et al., 1992a), a second messenger activated by 5-HT that plays an important role in producing both the short-term and the long-term changes in SNs (Kandel and Schwartz, 1982; Occor and Byrne, 1985; Schacher et al., 1988, 1991, 1993; Scholz and Byrne, 1988; Nazif et al., 1991; Ghirardi et al., 1992). Applications of 5-HT at the concentrations used here have been shown to increase the levels of cAMP and the activity of cAMP-dependent protein kinase

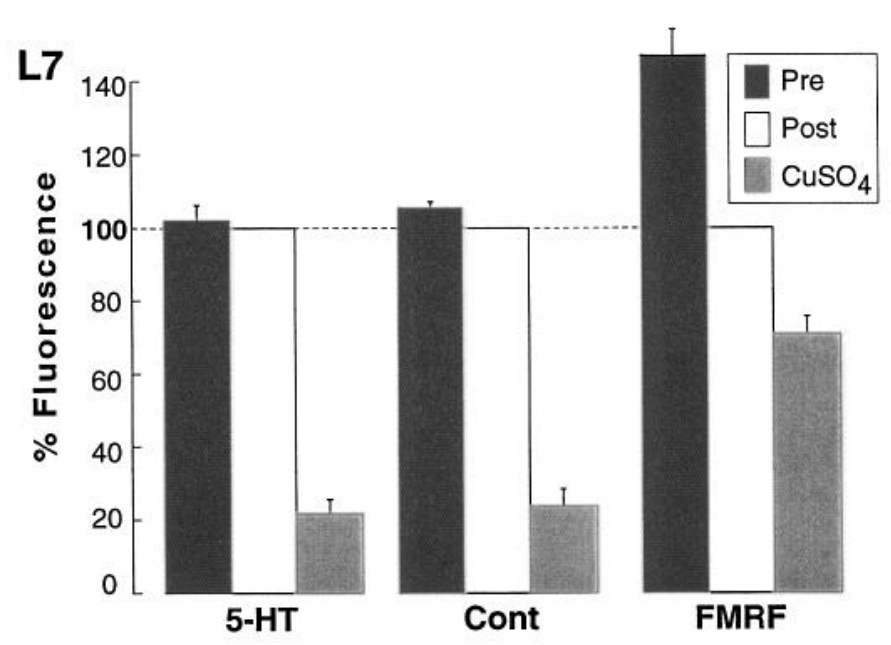

Figure 7. Changes in FITC-4E8-apCAM distribution on L7 neurites by FMRFamide. The bars are the mean \pm SEM of the fluorescent intensity of bound FITC-4E8 normalized for each cell to the level obtained after treatment (Post). An ANOVA indicated a significant effect of treatment on staining $(\mathrm{df}=2,12, F=24.621, p<0.001)$ and on level of fluorescence insensitive to quenching $(F=26.619, p<0.001)$. Multicomparison $t$ tests (Dunnett's $t$ ) indicated that treatment with FMRfamide had a significant effect on staining intensity $(t=5.824, p$ $<0.01)$ and the intensity of the component insensitive to quenching $(t$ $=6.779, p<0.01)$ compared to control. By contrast, treatment with 5 -HT had little effect on pixel intensity $(t=0.478, p>0.5)$ or the insensitive component $(t=0.767, p>0.5)$ compared to control.

$A$ in the cell bodies, growth cones, and neurites of SNs (Belardetti et al., 1986; Bacskai et al., 1993). A similar second messenger-mediated (e.g., cAMP-dependent) mechanism may underlie the FMRFamide-induced changes in motor neuron L7. FMRFamide evokes changes in ion conductances of some neurons via cyclic nucleotide-dependent modulation of ion channels (Colombaioni et al., 1985; Ichinose and McAdoo, 1988). Thus, as is the case for 5-HT actions on SNs that involve a number of long-term cellular and molecular changes induced by cAMP-dependent mechanisms, FMRFamide may evoke similar cAMP-dependent changes in the motor cell L7. Alternatively, the changes in apCAM distribution in L7 by FMRFamide may involve the participation of other intracellular signals/second messengers that only parallel the actions of cAMP on the SNs (e.g., Piomelli et al., 1987; Haydon et al., 1991). For both sensory and motor cells, however, the transmitter-induced changes in the distribution of apCAM may be regulated by second messenger-dependent modifications in cytoskeletal and other proteins involved in membrane cycling and turnover and/or the anchoring of cell adhesion molecules in the membrane (Werth et al., 1983; Forscher et al., 1987; Forscher, 1989; Matten et al., 1990; Sudhof and Jahn, 1991; Atashi et al., 1992; Lohof et al., 1992; Hu et al., 1993).

The cell-specific changes in growth cone behaviors induced by the transmitters correlate with the ability of the transmitters to modulate apCAM distribution on the surfaces of either SNs or L7. Thus, 5-HT applications alter SN growth cone-SN neurite interactions whereas FMRFamide applications alter L7 growth cone-L 7 neurite interactions. One interpretation of these results is that the change in growth cone behavior is linked causally to the change in the distribution of apCAM produced by the transmitters. This interpretation is supported by two observations. First, anti-apCAM antibodies or their Fab fragments, which do not by themselves alter membrane recycling (Bailey et al., 1992b), 
evoke similar changes in the behavior of the growth cones as those induced by the transmitters (see also Keller and Schacher, 1990). Second, some identified cells express little apCAM on their neurites and have identical types of growth cone behavior as those observed for cells expressing apCAM and treated with the transmitters or grown in the presence of anti-apCAM mAbs; they extend neurites that fail to fasciculate on each other (Flaster et al., 1986; Keller and Schacher, 1990). Consistent with this interpretation, the failure of the growth cones to fasciculate with homologous neurites when treated with transmitter is a consequence of the absence of homophilic interaction between the apCAM molecules on the growth cone and the neurite (Doherty and Walsh, 1989). Alternatively, the changes in growth cone behavior may arise directly from the transmitter-induced increase in membrane endocytosis or other actions at the growth cones or neurites that are evoked by the transmitters and/or their intracellular mediators (Haydon et al., 1984; Forscher et al., 1987; Masur et al., 1990; Kater and Mills, 1991; Lohof et al., 1992). Although the distribution of other surface molecules critical for growth cone motility and cell-cell interaction may be altered as a result of the increase in endocytosis, previous studies suggested a relative enrichment in the uptake of apCAM (Bailey et al., 1992b; Mayford et al., 1992). Moreover, the absence of other changes, such as the rate of growth cone extension on the polylysine-coated substrate, supports the idea that the reduced level of apCAM on the interacting membrane surfaces with the transmitters is contributing significantly to the change in the behavior of the growth cones.

Our results extend the reported observations of the role of specific transmitters or their receptors in modulating growth cone motility (Haydon et al., 1984), in establishing proper axon pathways and connections (Constantine-Paton et al., 1990), or in mediating long-term synaptic efficacy (Gustafsson and Wigstrom, 1988; Montarolo et al., 1988; Schacher et al., 1990). Thus activity-dependent release of specific neurotransmitters or neuropeptides (diffusible substances) during various stages in development, such as axon pathfinding, synapse formation, and/ or synapse stabilization, could alter the distribution of local cell surface substrate molecules and in turn affect the behavior of the invading growth cones or terminal endings. Because the changes in apCAM distribution are mediated by neurotransmitters that evoke opposite forms of long-term plasticity, it raises the possibility that the cell-specific actions of the two transmitters on apCAM distribution might contribute to the functional and structural changes at SN-L7 synapses accompanying long-term facilitation with 5 -HT and long-term inhibition with FMRFamide. In particular, the selective reduction of apCAM on the sensory cells by 5 -HT may decrease SN-SN contacts at extrasynaptic sites and facilitate interaction between the SNs and their follower cells leading to increases in the number of synaptic contacts. The reduction in apCAM on the main axon of the motor cell with applications of FMRFamide, on the other hand, may destabilize existing SN-L7 synaptic contacts and contribute to the loss of the synaptic contacts. Ihus, a number of activity-dependent forms of plasticity may utilize common mechanisms that differ only by the site of action of the critical intercellular signals and activation of the appropriate signal transduction machinery within the cells.

\section{References}

Atashi JR, Klinz SG, Ingraham CA, Matten WT, Schachner M, Maness PF (1992) Neural cell adhesion molecules modulate tyrosine phos- phorylation of tubulin in nerve growth cone membranes. Neuron 8:831-842.

Bacskai BJ, Hochner B, Mahaut-Smith M, Adams SR, Kaang B-K, Kandel ER, 'Isien RY (1993) Spatially resolved dynamics of cAMP and protein kinase A subunits in Aplysia sensory neurons. Science 260:222-226.

Bailey CH, Chen M, Kandel ER (1992a) Early steps in learning-related synaptic growth: cAMP simulates the heterologous endocytosis of apCAMs induced by 5-HT in sensory neurons of Aplysia. Soc Neurosci Abstr 18:941.

Bailey CH, Chen M, Keller F, Kandel ER (1992b) Serotonin-mediated endocytosis of apCAM: an early step of learning-related synaptic growth in Aplysia. Science 256:645-648.

Bailey CH, Montarolo PG, Chen M, Kandel ER, Schacher S (1992c) Inhibitors of protein and RNA synthesis block structural changes that accompany long-term heterosynaptic plasticity in Aplysia. Neuron 9:749-758.

Barzilai A, Kennedy TE, Sweatt JD, Kandel ER (1989) 5-HT modulates protein synthesis and the expression of specific proteins during long-term facilitation in Aplysia sensory neurons. Neuron 2:15771586 .

Belardetti F, Schacher S, Kandel ER, Siegelbaum SA (1986) The growth cones of Aplysia sensory neurons: modulation by serotonin of action potential duration and single potassium channel currents. Proc Natl Acad Sci USA 83:7094-7098.

Belardetti F, Kandel ER, Siegelbaum SA (1987) Neuronal inhibition by the peptide FMRF-amide involves opening of S-K ${ }^{+}$channels. Nature 325:153-156.

Bixby JL, Harris WA (1991) Molecular mechanisms of axon growth and guidance. Annu Rev Cell Biol 7:117-159.

Campanelli JT, Hoch W, Rupp F, Kreiner T, Sheller RH (1991) Agrin mediates cell contact-induced acetylcholine receptor clustering. Cell 67:909-916.

Caroni P, Schwab ME (1988) Two membrane protein fractions from rat central myelin with inhibitory properties for neurite growth and fibroblast spreading. J Cell Biol 106:1281-1288.

Colombaioni L, Paupardin-Tritsch D, Vidal PP, Gerschenfeld HM (1985) The neuropeptide FMRFamide decreases both the $\mathrm{Ca}^{++}$conductance and a cyclic 3'-5'-adenosine monophosphate-dependent $\mathrm{K}^{+}$ conductance in identified molluscan neurons. J Neurosci 5:25332538.

Constantine-Paton M, Cline HT, Debski E (1990) Patterned activity, synaptic convergence, and the NMDA receptor in developing visual pathways. Annu Rev Neurosci 13:129-154.

Cox EC, Muller B, Bonhoeffer F (1990) Axonal guidance in the chick visual system: posterior tectal membranes induce collapse of growth cones from the temporal retina. Neuron 2:31-37.

Doherty P, Walsh FS (1989) Neurite guidance molecules. Curr Opin Cell Biol 1:1102-1106.

Edelman GM, Cunningham BA (1991) Place-dependent cell adhesion, process retraction, and spatial signalling in neural morphogenesis. Cold Spring Harbor Symp Quant Biol 55:303-318.

Flaster MS, Ambron RT, Schacher S (1986) Growth cones isolated from identified Aplysia neurons in vitro: biochemical and morphological characterization. Dev Biol 118:557-586.

Forscher P (1989) Calcium and polyphosphoinositide control of cytoskeletal dynamics. Trends Neurosci 12:468-474.

Forscher P, Kaczmarek LK, Buchanan J, Smith SJ (1987) Cyclic AMP induces changes in distribution and transport of organelles within growth cones of Aplysia bag cell neurons. J Neurosci 7:3600-3611.

Fraser SE, Murray B, Chuong CM, Edelman GM (1984) Alteration of the retinotectal map in Xenopus by antibodies to neural cell adhesion molecules. Proc Natl Acad Sci USA 81:4222-4226.

Ghirardi M, Braha O, Hochner B, Montarolo PG, Kandel ER, Dale N (1992) Roles of PKA and PKC in facilitation of evoked and spontaneous transmitter release at depressed and non-depressed synapses in Aplysia sensory neurons. Neuron 9:479-489.

Glanzman DL, Mackey SL, Hawkins RD, Dyke AM, Lloyd PE, Kandel ER (1989) Depletion of serotonin in the nervous system of Aplysia reduces the behavioral enhancement of gill-withdrawal as well as the heterosynaptic facilitation produced by tail shock. J Neurosci 9:42004213.

Glanzman DL, Kandel ER, Schacher S (1991) Target-dependent morphological segregation of Aplysia sensory outgrowth in vitro. Neuron 7:903-913. 
Grenningloh G, Goodman CS (1992) Pathway recognition by neuronal growth cones: genetic analysis of neural cell adhesion molecules in Drosophila. Curr Opin Neurobiol 2:42-47.

Gustafsson B, Wigstrom H (1988) Physiological mechanisms underlying long-term potentiation. Trends Neurosci 11:156-162.

Hall 7W, Sanes JR (1993) Synaptic structure and development: the neuromuscular junction. Cell 72/Neuron 10[Suppl]:99-122.

Hawver DB, Schacher S (1993) Selective fasciculation as a mechanism for the formation of specific chemical connections between Aplysia neurons in vitro. J Neurobiol 24:368-383.

Haydon PG, McCobb DP, Kater SB (1984) Serotonin selectively inhibits growth cone motility and synaptogenesis of specific identified neurons. Science 226:561-564.

Haydon PG, Man-Song-Hing H, Doyle RT, Zoran M (1991) FMRFamide modulation of secretory machinery underlying presynaptic inhibition of synaptic transmission requires a pertussis-toxin sensitive G-protein. J Neurosci 11:3851-3860.

Hu Y, Barzilai A, Chen M, Bailey CH, Kandel ER (1993) 5-HT and cAMP induce the formation of coated pits and vesicles and increase the expression of clathrin light chain in sensory neurons of Aplysia. Neuron 10:921-929.

Hunter DD, Shah V, Merlie JP, Sanes JR (1989) A laminin-like adhesive protein concentrated in the synaptic cleft of the neuromuscular junction. Nature 338:229-234.

Ichinose M, McAdoo DJ (1988) The voltage-dependent, slow inward current induced by the neuropeptide FMR Famide in Aplysia neuron R14. J Neurosci 8:3891-3900.

Jessell TM (1988) Adhesion molecules and the hierarchy of neural development. Neuron 1:3-13.

Kandel ER, Schwartz JH (1982) Molecular biology of learning: modulation of transmitter release. Science 218:433-440.

Kater S, Mills L (1991) Regulation of growth cone behavior by calcium. J Neurosci 11:891-899.

Keller F, Schacher S (1990) Neuron-specific membrane glycoproteins promoting neurite fasciculation in Aplysia californica. J Cell Biol 111 : $2637-2650$.

Landmesser L, Dahm L, Tang J, Rutishauser U (1990) Polysialic acid as a regulator of intramuscular nerve branching during embryonic development. Neuron 4:655-667.

Lohof AM, Quillan M, Day Y, Poo M-M (1992) Asymmetric modulation of cytosolic cAMP activity induces growth cone turning. $J$ Neurosci 12:1253-1261.

Lumsden AGS, Davies AM (1983) Earliest sensory nerve fibers are guided to peripheral targets by attractants other than nerve growth factor. Nature 306:786-788.

Mackey SL, Kandel ER, Hawkins RD (1989) Identified serotonergic neurons LCB1 and RCB1 in the cerebral ganglia of Aplysia produce presynaptic facilitation of siphon sensory cells. J Neurosci 9:42274235 .

Masur SK, Kim YT, Wu CF (1990) Reversible inhibition of endocytosis in cultured neurons from the Drosophila temperature-sensitive mutant shibire. J Neurogenet 6:191-206.

Matten WT, Aubry M, West J, Maness PF (1990) Tubulin is phosphorylated at tyrosine by $\mathrm{pp}^{\text {rinc-ser }}$ in nerve growth cone membranes. J Cell Biol 111:1959-1970.

Mayford M, Barzilai A, Keller F, Schacher S, Kandel ER (1992) Modulation of an NCAM-related adhesion molecule with long-term synaptic plasticity in Aplysia. Science 256:638-644.

McMahan UJ (1991) The agrin hypothesis. Cold Spring Harbor Symp Quant Biol 55:407-418.

Montarolo PG, Goelet P, Castellucci VF, Morgan J, Kandel ER, Schacher S (1986) A critical period for macromolecular synthesis in longterm heterosynaptic facilitation in Aplysia. Science 234:1249-1254.

Montarolo PG, Kandel ER, Schacher S (1988) Long-term heterosynaptic inhibition in Aplysia. Nature 333:171-174.

Nazif FA, Byrne JH, Cleary LJ (1991) Cyclic AMP induces long-term morphological changes in sensory neurons. Brain Res 539:324-327.

Oakley RA, Tosncy KW (1991) Pcanut agglutinin and chondroitin6-sulphate are molecular markers for tissues that act as barriers to axon advance in the avian embryo. Dev Biol 147:187-206.

Occor KA, Byrne JH (1985) Membrane responses and changes in
cAMP levels in Aplysia neurons by serotonin, tryptamine, FMRFamide, and small cardioactive peptide (SCPb). Neurosci Lett 55:113118.

O'Leary DDM, Bicknese AR, De Carlos JA, Heffner CD, Koester SE, Kutka LJ, Terashima T (1991) Target selection by cortical axons alternative mechanisms to establish axonal projections in the developing brain. Cold Spring Harbor Symp Quant Biol 55:453-468.

Piomelli D, Volterra A, Dale N, Siegelbaum SA, Kandel ER, Schwartz JH, Belardetti F (1987) Lipoxygenase metabolites or arachidonic acid as second messengers for presynaptic inhibition of Aplysia sensory neurons. Nature 328:38-43.

Rajarathnam K, Hochman J, Schindler M, Ferguson-Miller S (1989) Synthesis, location, and lateral mobility of fluorescently labelled ubiquinone 10 in mitochondrial and artificial membranes. Biochem 28 : 3168-3176.

Raper JA, Kapfhammer JP (1990) The enrichment of a neuronal growth cone collapsing activity from embryonic chick brain. Neuron $4: 21-29$.

Rayport SC, Schacher S (1986) Synaptic plasticity in vitro: cell culture of identified Aplysia neurons mediating short-term habituation and sensitization. J Neurosci 6:759-763.

Reichardt LF, Tomaselli KJ (1991) Extracellular matrix molecules and their receptors: functions in neural development. Annu Rev Neurosci 14:531-570.

Rutishauser U, Acheson A, Hall A, Mann D, Sunshine J (1988) The neural cell adhesion molecule (NCAM) has a regulator of cell-cell interactions. Science 240:53-57.

Schacher S, Montarolo PG (1991) Target-dependent structural changes in sensory neurons of Aplysia accompany long-term heterosynaptic inhibition. Neuron 6:679-690.

Schacher S, Prohansky E (1983) Neurite regeneration by Aplysia neurons in dissociated cell culture: modulation by Aplysia hemolymph and the presence of the initial axonal segment. J Neurosci 3:24032413.

Schacher S, Castellucci VF, Kandel ER (1988) Cyclic AMP evokes long-term facilitation in Aplysia sensory neurons that requires new protein synthesis. Science 240:1667-1669.

Schacher S, Montarolo PG, Kandel ER (1990) Selective short- and long-term effects of serotonin, small cardioactive peptide, and tetanic stimulation on sensorimotor synapses of Aplysia in culture. J Neurosci 10:3286-3294.

Schacher S, Glanzman DL, Barzilai A, Dash D, Grant SGN, Keller F, Mayford M, Kandel ER (1991) Long-term facilitation in Aplysia: persistent phosphorylation and structural changes. Cold Spring Harbor Symp Quant Biol 55:187-202.

Schacher S, Kandel ER, Montarolo PG (1993) cAMP and arachidonic acid simulate long-term structural and functional changes produced by neurotransmitters in Aplysia sensory neurons. Neuron 10:10791088 .

Scholz KP, Byrne JH (1988) Intracellular injection of cAMP induces a long-term reduction of neuronal potassium currents. Science 240 1664-1667.

Schwab ME, Kapfhammer JP, Bandtlow LE (1993) Inhibitors of neurite growth. Annu Rev Neurosci 16:565-596.

Sudhof TC, Jahn R (1991) Proteins of synaptic vesicles involved in exocytosis and membrane recycling. Neuron 6:665-677.

Takeichi M (1991) Cadherin cell adhesion receptors as a morphogenetic regulator. Science 251:1451-1455.

Tang J, Landmesser L, Rutishauser U (1992) Polysialic acid influences specific pathfinding by avian motoneurons. Neuron 8:1031-1044.

Tessier-Lavigne M, Placzek M, Lumsden AGS, Dodd J, Jessell TM (1988) Chemotropic guidance of developing axons in the mammalian central nervous system. Nature 336:775-778.

Usdin TB, Fischbach GD (1986) Purification and characterization of a polypeptide from chick brain that promotes the accumulation of acetylcholine receptors in chick myotubes. J Cell Biol 103:493-507.

Werth DK, Niedel JE, Pastan I (1983) Vinculin, a cytoskeletal substrate of protein kinase C. J Biol Chem 258:11423-11429.

Zhang ZS, Fang B, Marshak DW, Byrne JH, Cleary LJ (1991) Serotonergic varicosities make synaptic contacts with pleural sensory neurons of Aplysia. J Comp Neurol 311:259-270. 